\title{
Complex aortic arch surgery: Back to the future?
}

\author{
Friedhelm Beyersdorf, MD, PhD
}

\author{
From the Department of Cardiovascular Surgery University Heart Center Freiburg, Freiburg, Germany. \\ Disclosures: Author has nothing to disclose with regard to commercial support. \\ Received for publication April 4, 2016; accepted for publication April 4, 2016. \\ Address for reprints: Friedhelm Beyersdorf, MD, PhD, Department of Cardiovascular Surgery, University Heart \\ Center Freiburg, Hugstetterstrasse 55, D-79106, Freiburg, Germany (E-mail: friedhelm.beyersdorf@ \\ uniklinik-freiburg.de). \\ J Thorac Cardiovasc Surg 2016;152:7-8 \\ $0022-5223 / \$ 36.00$ \\ Copyright $(2016$ by The American Association for Thoracic Surgery \\ http://dx.doi.org/10.1016/j.jtcvs.2016.04.015
}

The armamentarium of surgeons nowadays includes a wide spectrum of methods, ranging from conventional open procedures to minimally invasive, endovascular, hybrid, and catheter-based procedures. Many of these approaches were developed by surgeons, and they are used more and more for the benefit of our patients.

In general, less invasive or hybrid procedures for the aortic arch are seen as safer options than the open, conventional method. In previous publications, conventional aortic arch surgery was among the most complex cardiac procedures, with high mortality and morbidity rate, and every effort was done to reduce these complications, mainly by developing minimally invasive, hybrid procedures ${ }^{1}$ or endovascular total arch repair. ${ }^{2}$

Creative surgeons are not just developing new and less invasive procedures, however, they are also working on improving existing, conventional methods. Total arch replacement with the Thoraflex hybrid frozen elephant trunk (FET) prosthesis (VASCUTEK, a TERUMO Company, Inchinnan, UK) is an example in which a conventional method, which was inferior for quite some time to newer less invasive procedures, is now showing better and improved short-term and midterm results relative to less invasive (debranching) techniques.

With the development of the FET procedure ${ }^{3}$ (E-vita OPEN PLUS; JOTEC GmbH, Hechingen, Germany), new and exciting options evolved for this kind of surgery. Combining FET with a 4-branched arch graft for complex aortic arch pathology (Thoraflex) has further expanded the surgical possibilities for all arch pathologies.

In this respect, the article in this issue of the Journal from Shrestha and colleagues ${ }^{4}$ is very exciting. It shows that open, conventional surgery for the aortic arch could be performed with excellent early and midterm results in the first 100 patients during a 4-year period.

Shrestha and colleagues ${ }^{4}$ report a 30-day mortality of 7\% and an $81 \%$ survival after a mean follow-up of 3.1 years. The Thoraflex prosthesis is technically relatively easy to implant, and the availability of the sewing collar for the distal anastomosis transforms this formerly very demanding technical part of the aortic arch replacement into a pleasant

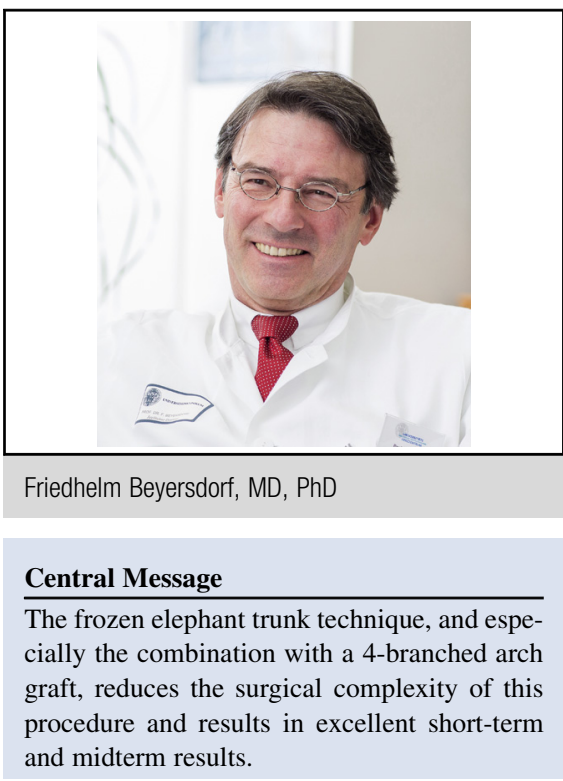

See Article page 148.

See Editorial Commentary page 160.

surgical challenge. Different sizes are available for the unstented and stented portion to fit all pathologies. Especially in patients with malperfusion, FET with a 4-branched arch prosthesis allows very good results relative to previous methods. ${ }^{4}$

Interestingly, Shrestha and colleagues ${ }^{4}$ have shown that even in patients with chronic type A dissection (CAD), Thoraflex FET implantation resulted in an increase of the true lumen diameter and a decrease of the false lumen diameter in the stented segment A. This shows that in chronic dissection the true lumen can still be enlarged and the false lumen able to decrease its diameter. Likewise intriguing are the aortic diameters in segment $\mathrm{A}$, which were significantly smaller in acute type A dissection (AAD) and patients with chronic type A dissection or aneurysm, a finding that is in line with the recent publication by Rylski and colleagues. ${ }^{5}$ Nevertheless, the number of secondary reinterventions on the downstream aorta, especially in patients with aneurysm and chronic type A dissection, underscores again the importance of a lifelong follow-up of these patients.

The well described "beating heart aortic arch surgery" technique ${ }^{6}$ now allows even the combination of complex aortic root procedures in younger patients with complex aortic arch and proximal descending aortic replacements. 
One of the devastating complications after arch and descending aortic surgery is paraplegia. Spinal cord injury is reported, with an incidence as great as $20 \%$ in some series. In the study in this issue, however, Shrestha and colleagues ${ }^{4}$ report only a $7 \%$ incidence of paraparesis. They attribute this good result to the fact that they have used only short FET $(100 \mathrm{~mm})$, cerebrospinal fluid drainage in all elective cases (whereas patients with AAD receive a cerebrospinal fluid drain as soon as possible after surgery), a core temperature lower than $25^{\circ} \mathrm{C}$, and a short duration of hypothermic circulatory arrest in all cases. This again underscores the importance of cold $\left(<25^{\circ} \mathrm{C}\right)$ perfusion of the lower body and warns against reports with "warm body perfusion" during aortic arch replacements.

Even so, the incidence of recurrent nerve palsy in $25 \%$ of all patients was rather high. To reduce this complication, at my center we almost always perform the distal aortic anastomosis between the left carotid artery and the origin of the left subclavian artery. We cut the subclavian artery from the distal aortic arch and oversew this defect with a double running polypropylene monofilament suture, with felt added if necessary. The distal aortic anastomosis is then performed, followed by anastomosing the third branch of the Thoraflex prosthesis to the subclavian artery. We then start rewarming the lower body and the left subclavian artery by perfusing through the fourth branch of the arch graft.

The reported stroke rates of $9 \%$ overall and $14 \%$ in patients with AAD (5/37) are very low, and Shrestha and colleagues ${ }^{4}$ are to be congratulated for these very good results. An easy trick to wash out air, carbon dioxide, or debris from the supra-aortic branches is to perfuse first only 1 carotid artery for a short time (1-2 minutes) while keeping the contralateral carotid artery open, and then proceed vice versa. It is astonishing to see in some cases the amount of air and solid material that is washed out by this maneuver.
Still unanswered is the question of what to do in uncomplicated AAD. Should one proceed with the FET technique or go ahead with limited repair of AAD (ie, supracoronary ascending replacement)? The decision depends of course on the age, the overall morbidity, and the extent of the disease of the patient. Nevertheless, only further studies ${ }^{7-10}$ will shed light on this question.

\section{References}

1. Chen LW, Wu XJ, Dai XF, Liao DS, Hu YN, Zhang H, et al. Repair of acute type A aortic dissection with ascending aorta replacement combined with open fenestrated stent graft placement. Ann Thorac Surg. 2016;101:644-9.

2. Katada Y, Kondo S, Tsuboi E, Rokkaku K, Irie Y, Yokoyama H. Endovascular total arch repair using in situ fenestration for arch aneurysm and chronic type A dissection. Ann Thorac Surg. 2016;101:625-30.

3. Leontyev S, Tsagakis K, Pacini D, Di Bartolomeo R, Mohr FW, Weiss G, et al. Impact of clinical factors and surgical techniques on early outcome of patients treated with frozen elephant trunk technique by using EVITA open stent-graft: results of a multicentre study. Eur J Cardiothorac Surg. 2016;49:660-6.

4. Shrestha M, Kaufeld T, Beckmann E, Fleissner F, Umminger J, Abd Alhadi F, et al. Total aortic arch replacement with the Thoraflex hybrid frozen elephant trunk prosthesis: single-center results of the first 100 patients. J Thorac Cardiovasc Surg. 2016;152:148-59.

5. Rylski B, Blanke P, Beyersdorf F, Desai ND, Milewski RK, Siepe M, et al. How does the ascending aorta geometry change when it dissects? J Am Coll Cardiol. 2014;63:1311-9.

6. Martens A, Koigeldiyev N, Beckmann E, Fleissner F, Kaufeld T, Krueger H, et al. Do not leave the heart arrested. Non-cardioplegic continuous myocardial perfusion during complex aortic arch repair improves cardiac outcome. Eur J Cardiothorac Surg. 2016;49:141-8.

7. Di Eusanio M, Berretta P, Cefarelli M, Castrovonci S, Folesani G, Alfonsi J, et al Long-term outcomes after aortic arch surgery: results of a study involving 623 patients. Eur J Cardiothorac Surg. 2015;48:483-90.

8. Shi E, Gu T, Yu Y, Yu L, Wang C, Fang Q, et al. Early and midterm outcomes of hemiarch replacement combined with stented elephant trunk in the management of acute DeBakey type I aortic dissection: comparison with total arch replacement. J Thorac Cardiovasc Surg. 2014;148:2125-31.

9. Omura A, Miyahara S, Yamanaka K, Sakamoto T, Matsumori M, Okada K, et al. Early and late outcomes of repaired acute DeBakey type I aortic dissection after graft replacement. J Thorac Cardiovasc Surg. 2016;151:341-8.

10. Shrestha M, Bachet J, Bavaria J, Carrel T, De Paulis R, Di Bartolomeo R, et al. Current status and recommendations for use of frozen elephant trunk technique: a position paper by the Vascular Domain of EACTS. Eur J Cardiothorac Surg. 2015;47:759-69. 